\title{
Digestibilidad in situ de rastrojo de maíz tratado con enzimas fibrolíticas
}

\section{In situ digestibility of corn stover' treated with fibrolytic enzymes}

Fecha de recepción: 1 de abril de 2016

Fecha de aprobación: 2 de diciembre de 2016
Hugo de Jesús López-Inzunza ${ }^{1}$ Bertha Bienvenida Chongo-García ${ }^{2}$

Orestes La O-León ${ }^{3}$ Juan Eulogio Guerra-Liera ${ }^{4}$ Maribel Luna-López ${ }^{5}$ Samuel Jesús Castro-Camacho ${ }^{6}$ Luciano Abelino López-Juárez ${ }^{7}$

\section{Resumen}

Se estudió la digestibilidad in situ de la matriz fibrosa de rastrojo de maíz tratado con un complejo enzimático, utilizando dos toros suizos (700 kg PV) con cánula ruminal, en un diseño de bloques al azar, donde cada animal constituyó un bloque, y los tratamientos fueron tres niveles de enzimas: $0 \mathrm{~g}$ (TC), 1 $\mathrm{g}$ (T1) y $2 \mathrm{~g}$ (T2) de Fibrozyme ${ }^{\circledR} \mathrm{kg}$ forraje $\mathrm{e}^{-1} \mathrm{MS}$. Las muestras (5 g de materia seca en bolsas de nailon) se incubaron en el rumen, y se extrajeron a las 6, 12, 24, 48 y 72 horas posfermentación. La degradabilidad se analizó mediante regresión no lineal, con la utilización del modelo $\mathrm{P}=\mathrm{a}+\mathrm{b}^{*}(1-\mathrm{e}-\mathrm{c} *(\mathrm{t}-\mathrm{to}))$. La porción soluble de la matriz fibrosa, determinada por lavado de las bolsas, resultó similar en ambas fracciones de fibra (FDN y FDA), y fue de $0.50,0.59$ y $0.68 \%$ para sus tres tratamientos, respectivamente. Los valores de degradabilidad de la fracción insoluble, pero degradable, fueron: 37.28, 36.64 y $37.30 \%$ para FDN y $33.85,35.07$ y $41.19 \%$ para la FDA, con sus respectivos tratamientos. El T2 (FDN) y el TC (FDA) registraron los mayores valores de velocidad de degradación (c \%/h). La fase Lag fue, aproximadamente, de $3.80,4.90$ y $4.80 \mathrm{~h}$ para FDN y $5.30,5.30$ y $4.90 \mathrm{~h}$ para la FDA, con sus tres respectivos tratamientos. La degradabilidad efectiva (DE) de la FDN osciló entre 19.70-14.40, 21.30-15.90 y 22.10-16.60 \% y la de la FDA, entre 20.30-15.30, 21.10-15.80 y 23.40-17.30 \%, en cada uno de los tratamientos, al utilizar diferentes constantes de recambio ruminal. El rastrojo de maíz mostró buen potencial de degradación,

1 Universidad Autónoma de Sinaloa (Culiacán de Rosales, Sinaloa, México).

2 Ph. D. Instituto de Ciencia Animal (Mayabeque, Cuba). bchongo@ica.co.cu.

Ph. D. Instituto de Ciencia Animal (Mayabeque, Cuba). olao@ica.co.cu.

Ph. D. Universidad Autónoma de Sinaloa (Culiacán de Rosales, Sinaloa, México).

Universidad Autónoma de Sinaloa (Culiacán de Rosales, Sinaloa, México). maribelluna@uas.edu.mx.

6 Universidad Autónoma de Sinaloa, (Culiacán de Rosales, Sinaloa, México).

7 Universidad Autónoma de Sinaloa, (Culiacán de Rosales, Sinaloa, México). 
superior al $39 \%$ en FDN y de $44 \%$ en FDA, cuando fue tratado con los niveles más altos de enzimas fibrolíticas (T2). Los resultados demostraron que puede hacerse un uso óptimo de la matriz fibrosa como fuente energética para el animal cuando se utilizan complejos enzimáticos en esquilmos agrícolas como el rastrojo de maíz mediante su fermentación ruminal.

Palabras clave: digestibilidad in situ; enzimas fibrolíticas; nutrientes; rastrojo de maíz.

\begin{abstract}
In situ digestibility of corn stover fiber matrix treated with an enzymatic complex was studied by using two Swiss bulls (700 kg PV) with ruminant cannula. We used a randomized block design, where each animal constituted a block, and three enzyme levels: $0 \mathrm{~g}$ (TC), $1 \mathrm{~g}$ (T1), and $2 \mathrm{~g}$ (T2) of Fibrozyme ${ }^{\circledR} \mathrm{kg}_{\text {fodder }}{ }^{-1}$ MS. The samples ( $5 \mathrm{~g}$ of dry matter in nylon bags) were incubated in the ruminant, and were extracted at $6,12,24,48$ and 72 hours after fermentation.
\end{abstract}

Debasement was analyzed by a none lineal regression, using the $\mathrm{P}=\mathrm{a}+\mathrm{b}^{*}\left(1-\mathrm{e}-\mathrm{c}^{*}\right.$ (t-to)) model. The soluble portion of the fibrous matrix, determined by washing the bags, resulted similar in both fiber fractions (FDN and FDA), with values of $0.50,0.59$ and $0.68 \%$ respectively for each treatment. The degradability values of the insolvent, but degradable, fraction were: $37.28,36.64$ and $37.30 \%$ for FDN, and 33.85, 35.07 and $41.19 \%$ for FDA, with their three respective treatments.

The T2 (FDN) and the TC (FDA) registered the highest degradation speed values (c $\% / h)$. The lag phase was approximately of 3.80, 4.90 and $4.80 \mathrm{~h}$ for FDN and 5.30, 5.30 and $4.90 \mathrm{~h}$ for FDA, with their three respective treatments. The FDN effective degradability (DE) oscillated between 19.70-14.40, 21.30-15.90 and 22.10-16.60 \%; and the FDA, between 20.30-15.30, 21.10-15.80 and 23.40-17.30\%, in each one of the treatments, by using different ruminant exchange constants.

The corn stover showed good degradation potential, over $39 \%$ in FDN and $44 \%$ in FDA, when treated with the highest levels of fibrolytic enzymes (T2). The results showed that an optimal usage of the fiber-matrix as energy source for the animal can be achieved, when enzymatic complexes are used in agriculture products and residues such as corn stover through its ruminal fermentation.

Keywords: corn stover; fibrolytic enzymes; in situ digestibility; nutrients.

\title{
Cómo citar este artículo:
}

López-Inzunza G, Chongo-García BB, La-O-León O, Guerra-Liera JE, Luna-López M, Castro-Camacho SJ, López-Juárez LA. Digestibilidad in situ de rastrojo de maíz tratado con enzimas fibrolíticas. Rev. Cien. Agri. 2017; 14(1): 31-37. 


\section{Introducción}

El rastrojo de maíz es el alimento más abundante para los rumiantes durante parte del año en muchas regiones de México, pero su aporte nutricional es insuficiente para mantenerlos. La producción de rastrojos está asociada directamente con la de granos, por lo que a medida que aumenta la cantidad producida de granos para satisfacer la demanda alimenticia de la población, se incrementa la disponibilidad de estos residuos (1). Según la Secretaría de Agricultura, Ganadería, Desarrollo Rural, Pesca y Alimentación de México (2), la producción nacional de esquilmos agrícolas en este país es de 45 millones de toneladas de MS, provenientes de diez cultivos, de los cuales los más importantes son el maíz, el sorgo, el trigo y la cebada.

Los esquilmos agrícolas son una fuente de energía abundante y barata para los rumiantes, por lo que se ha intentado mejorar su digestibilidad a través de procesos físicos, químicos y biológicos o mediante aditivos, como cultivos microbianos y enzimas fibrolíticas exógenas (3).

Las enzimas fibrolíticas exógenas pueden ser una alternativa para aprovechar los nutrientes potencialmente digestibles de estos esquilmos. Los componentes primarios de las paredes celulares de los forrajes son la celulosa y la hemicelulosa, los cuales son digeridos por las enzimas fibrolíticas de las bacterias y protozoarios ruminales (4). Aunque este proceso es eficiente, se buscan métodos que mejoren la digestión de la fibra por el ganado, como la adición de celulasas y xilanasas que complementen la actividad celulolítica de las bacterias ruminales (5).

Por lo tanto, el objetivo de este trabajo fue evaluar el efecto del complejo enzimático Fibrozyme ${ }^{\circledR}$ (Alltech INC, Nicholasville, KY, USA) sobre la degradabilidad ruminal in situ de las fracciones de fibra (FDN y FDA) de rastrojo de maíz con diferentes tratamientos $\left(0,1\right.$ y 2 g de Fibrozyme ${ }^{\circledR} \mathrm{kg}^{-1}$ forraje MS). Para el ajuste de los parámetros del modelo se utilizó el programa NEWAY EXCEL versión 5.0 WINDOWS ${ }^{\circledR}(6)$, el cual permite fijar dichos parámetros.

\section{Materiales y métodos}

La presente investigación se llevó a cabo en la posta zootécnica y en el laboratorio de bromatología y nutrición animal de la Facultad de Agronomía de la Universidad Autónoma de Sinaloa, México (FAUAS), localizada en el km 17.5 de la carretera Culiacán-El Dorado, ubicada en la longitud $107^{\circ}$ $24^{\prime}$ y latitud $24^{\circ} 49^{\prime}$ norte a una altura de 62 msnm y con una temperatura media anual de 24.9 ${ }^{\circ} \mathrm{C}$ (mínima en el mes de enero, con $19.4^{\circ} \mathrm{C}$, y máxima los meses de abril y mayo, con $29.3^{\circ} \mathrm{C}$ ). El clima se define como cálido y semiseco, con una precipitación anual de $671.7 \mathrm{~mm}$, con 203.1 mm como máxima para el mes de agosto, y abril y mayo con el menor índice, correspondiente a 1.6 $\mathrm{mm}$ (7).

Para estudiar la digestibilidad ruminal in situ de la fracción fibrosa (DISFDN y DISFDA), se recolectaron muestras al azar, simulando el ramoneo del animal (8), de pacas de rastrojo de maíz procedentes de la Unión Ganadera Regional de Sinaloa (UGRS). Las muestras recolectadas de todas las pacas se homogeneizaron para obtener un pool de muestra representativo. El material se secó en estufas de aire forzado a $60{ }^{\circ} \mathrm{C}$ durante $48 \mathrm{~h}$; luego, se molió a un tamaño de partícula de $1 \mathrm{~mm}$ y se le determinó la composición química. El resto de material se molió a un tamaño de partícula de 2 $\mathrm{mm}$ y se utilizó para la prueba de digestibilidad de los componentes estudiados.

Para los estudios in situ se utilizaron dos toros suizos puros (bos taurus), con un peso vivo (PV) aproximado de $700 \mathrm{~kg}$, canulados en rumen, en un diseño de bloques al azar, donde cada animal constituyó un bloque, y los tratamientos fueron tres niveles de enzima $\left(0,1\right.$ y $2 \mathrm{~g}$ de Fibrozyme ${ }^{\circledR}$ $\mathrm{kg}^{-1}$ forraje MS). Los animales se alimentaron con $4 \mathrm{~kg}$ de concentrado/animal/día, $5 \mathrm{~g}$ de sales minerales/animal/día y rastrojo de maíz y agua ad libitum para garantizar sus requerimientos de mantenimiento y la actividad celulolítica ruminal. Los días de adaptación a la dieta fueron 14, más 3 de muestreo. Se alimentó a los bovinos a las 8:00 y $17: 00 \mathrm{~h}$. 
Se emplearon bolsas de nailon de $15.0 \times 7.5 \mathrm{~cm}$; tamaño de poro $52 \pm 10 \mu \mathrm{m}$ con $5 \mathrm{~g}$ de materia seca (MS) de muestra previamente numeradas y taradas. Se colocaron 3 bolsas/tratamiento/animal dentro del rumen por $6,12,24,48$ y $72 \mathrm{~h}$, y se retiraron en orden inverso.

Luego, se lavaron por fuera con agua corriente hasta que el líquido fue claro, con la finalidad de eliminar el material contaminante y soluble. Las bolsas se secaron en una estufa de aire forzado a $60{ }^{\circ} \mathrm{C}$ durante 48 h. Se estimó el contenido por triplicado de FDN y FDA sobre el contenido residual. La degradabilidad ruminal in situ se determinó como el porcentaje de desaparición en la bolsa de FDN y FDA después de la incubación. La solubilidad de la hora cero se obtuvo al incubar las bolsas en agua a $37^{\circ} \mathrm{C}$, durante $15 \mathrm{~min}$.

La DISFDN y DISFDA se determinaron por el método de McDonald (9), donde:

$\mathrm{P}=\mathrm{a}+\mathrm{b}(1-\mathrm{e}-\mathrm{c}(\mathrm{t}-\mathrm{to}))$ para $\mathrm{t}>$ to

$\mathrm{a}=$ fracción soluble

$b=$ fracción degradable si el tiempo no es limitante

$\mathrm{c}=$ velocidad de degradación de b

$\mathrm{a}+\mathrm{b}=$ potencial de degradación

Este modelo permite calcular el tiempo Lag (h), que es el tiempo o fase que necesitan los microorganismos para colonizar la fibra.

Como los valores de degradabilidad obtenidos a partir de la técnica de degradabilidad ruminal in situ podrían no reflejar lo que ocurre en condiciones normales de alimentación si estos resultados no son corregidos por la velocidad de pasaje, se estimó la degradabilidad efectiva de la FDN y FDA, o sea la cantidad que es degradada cuando el alimento permanece en el rumen por un tiempo determinado. Para ello se asumieron distintas tasas de pasaje (kp): 3, 4 y $5 \%$ hora $^{-1}$. Para esto se utilizó la ecuación $\mathrm{DE}=\mathrm{a}+((\mathrm{b} \mathrm{c}) /(\mathrm{c}+\mathrm{k}))(10)$. La determinación de las pérdidas por lavado o fracción soluble fue según Pedraza (11).
Se realizó el análisis químico de materia seca (MS), cenizas (Cza) y proteína bruta (PB) conforme a la AOAC (12), la fibra detergente neutro (FDN) y la fibra detergente ácido (FDA) se analizaron de acuerdo con Van Soest (13).

Para el ajuste de los parámetros del modelo se utilizó el programa NEWAY EXCEL versión 5.0 WINDOWS ${ }^{\circledR}(6)$, el cual permite fijar dichos parámetros. Se utilizó un diseño experimental de bloques completamente al azar, donde cada animal (toro) constituyó un bloque, y los tratamientos fueron tres niveles de enzima $\left(0,1\right.$ y 2 g Fibrozyme $^{\circledR}$ $\mathrm{kg}^{-1}$ forraje MS). Las variables fueron analizadas mediante análisis de varianza. Para la diferencia múltiple de medias se utilizó la prueba de Tukey usando el PROC NLIN de SAS/STAT ${ }^{\circledR}$ v. 9.2 (SAS Institute, 2010).

\section{Resultados y discusión}

Las Tablas I y II muestran la composición química y las características de la degradabilidad de los componentes estudiados. La fracción soluble de la FDN aumentó el $18 \%$ y el $36 \%$ con el T1 y el T2, respectivamente. Bourgetts et al. (14) reportaron aumentos inferiores (17 y $5 \%$ ) en la fracción soluble utilizando subproductos de maíz tratados con álcalis ( $\mathrm{NaOH}$ y urea). El valor de la fracción degradable (b) de la FDN aumentó ligeramente con el T2 y se redujo $1.71 \%$ con el T1. Aumentos superiores $(74 \%)$ fueron reportados por Elizondo et al. (15) al evaluar un tratamiento alcalino (urea) sobre la calidad nutricional de esquilmos agrícolas de maíz. La velocidad de degradación (c) aumentó el $38.78 \%$ y el $44.14 \%$ con el T1 y el T2, respectivamente.

Al respecto, diferentes trabajos coinciden en la gran variabilidad existente en las características de la degradabilidad ruminal de los nutrientes de diferentes esquilmos agrícolas, entre los que se encuentra el rastrojo de maíz (16).

En general, la FDN se comportó adecuadamente en la desaparición de la fracción degradable (b), si se tiene en cuenta que para iniciar con la degradación debe existir primero una colonización de la fibra por la microbiota ruminal. 
Tabla I. Composición química (\%) del esquilmo agrícola estudiado.

\begin{tabular}{|c|c|}
\hline Parámetro & Rastrojo de maíz \\
\hline MS (\%) & 89.25 \\
\hline Cza (\%) & 7.59 \\
\hline PC (\%) & 4.62 \\
\hline FDN (\%) & 72 \\
\hline FDA (\%) & 46.75 \\
\hline
\end{tabular}

MS = materia seca; Cza = ceniza; $\mathrm{PC}=$ proteína cruda; $\mathrm{FDN}=$ fibra detergente ácido; FDA = fibra detergente ácido.

Tabla II. Degradabilidad ruminal in situ de FDN y FDA (\%) de esquilmo de maíz con diferentes tratamientos de fibrozyme.

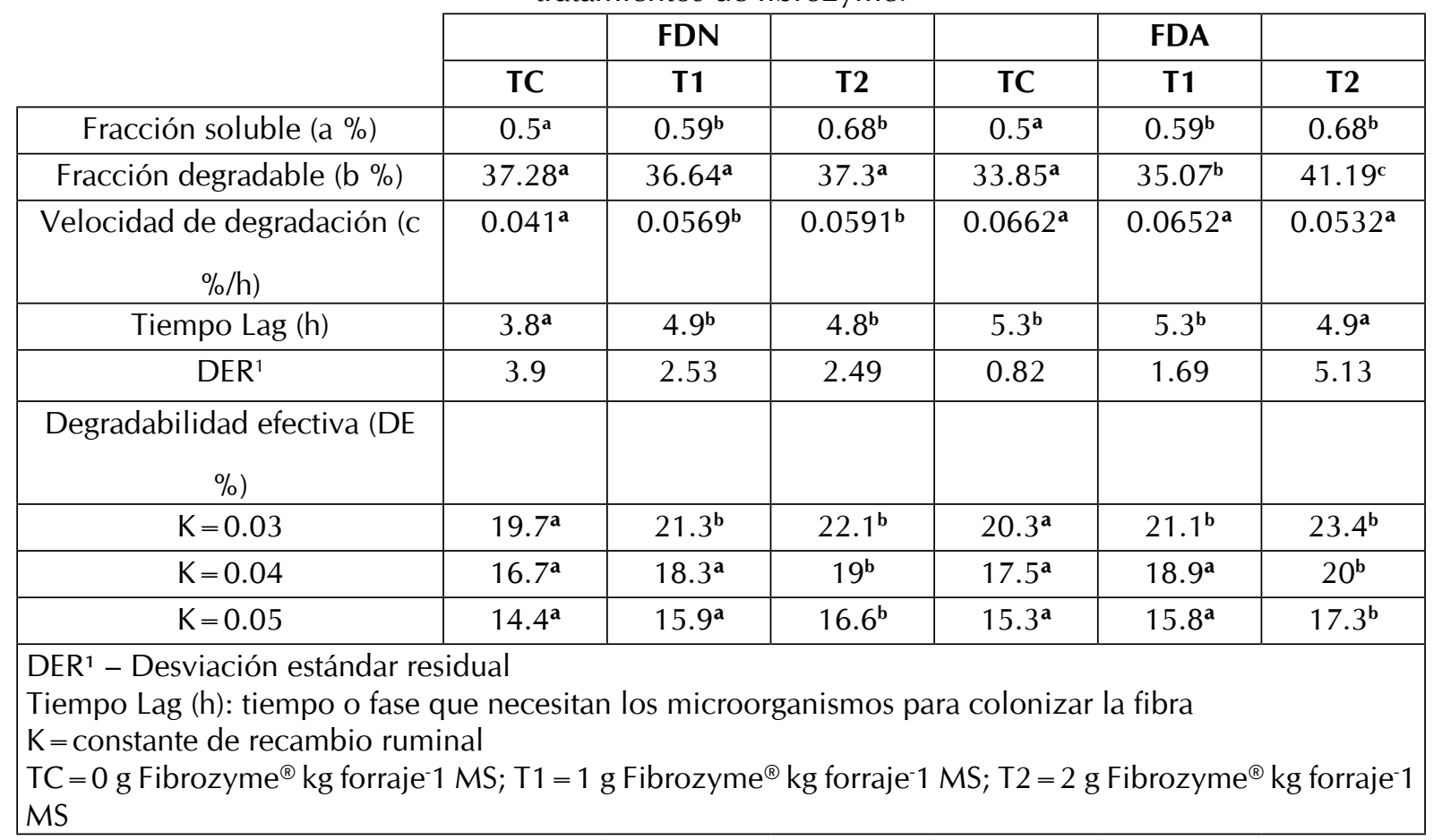

La fracción soluble (a) de la FDA tuvo un comportamiento similar a la de la FDN. La fracción degradable (b) aumentó el $3.60 \%$ con el T1, resultando inferior a los valores informados por Bourgetts (14) en rastrojo de maíz con $\mathrm{NaOH}$, mientras que con el T2 la fracción degradable logró un aumento de $21.68 \%$, superior al encontrado por Elizondo et al. (15) en rastrojo de maíz tratado con urea. La velocidad de degradación disminuyó con ambos tratamientos (T1 y T2), respecto al tratamiento control (TC). Una disminución similar en la constante de degradación fue encontrada por Yescas et al. (17) al tratar rastrojo de maíz con enzimas fibrolíticas respecto al testigo.
La fase lag de la FDN fue menor que la de la FDA para los tres tratamientos (TC, $\mathrm{T} 1$ y $\mathrm{T} 2$ ), lo que se asocia a la pobre acción que tienen los microorganismos ruminales en la degradación del complejo lignocelulósico.

Respecto a las degradabilidades efectivas, los valores oscilaron entre 19.70-14.40 (TC), 21.30-15.90 (T1) y $22.10-16.60 \%$ (T2) para FDN, y 20.30-15.30 (TC), $21.10-15.80$ (T1) y $23.40-17.30 \%$ (T2) para la FDA, con diferentes constantes de recambio ruminal (K). Los resultados fueron inferiores a los de Salado et al. (18) para la degradabilidad efectiva de la FDN en cascarilla de soja y afrechillo de trigo. 
La desaparición de las fracciones de fibra (FDN y FDA), en función del tiempo de incubación ruminal (gráficas 1 y 2), mostró un aumento exponen- cial $(\mathrm{P}<0.01)$ en el tiempo hasta el último horario de incubación.

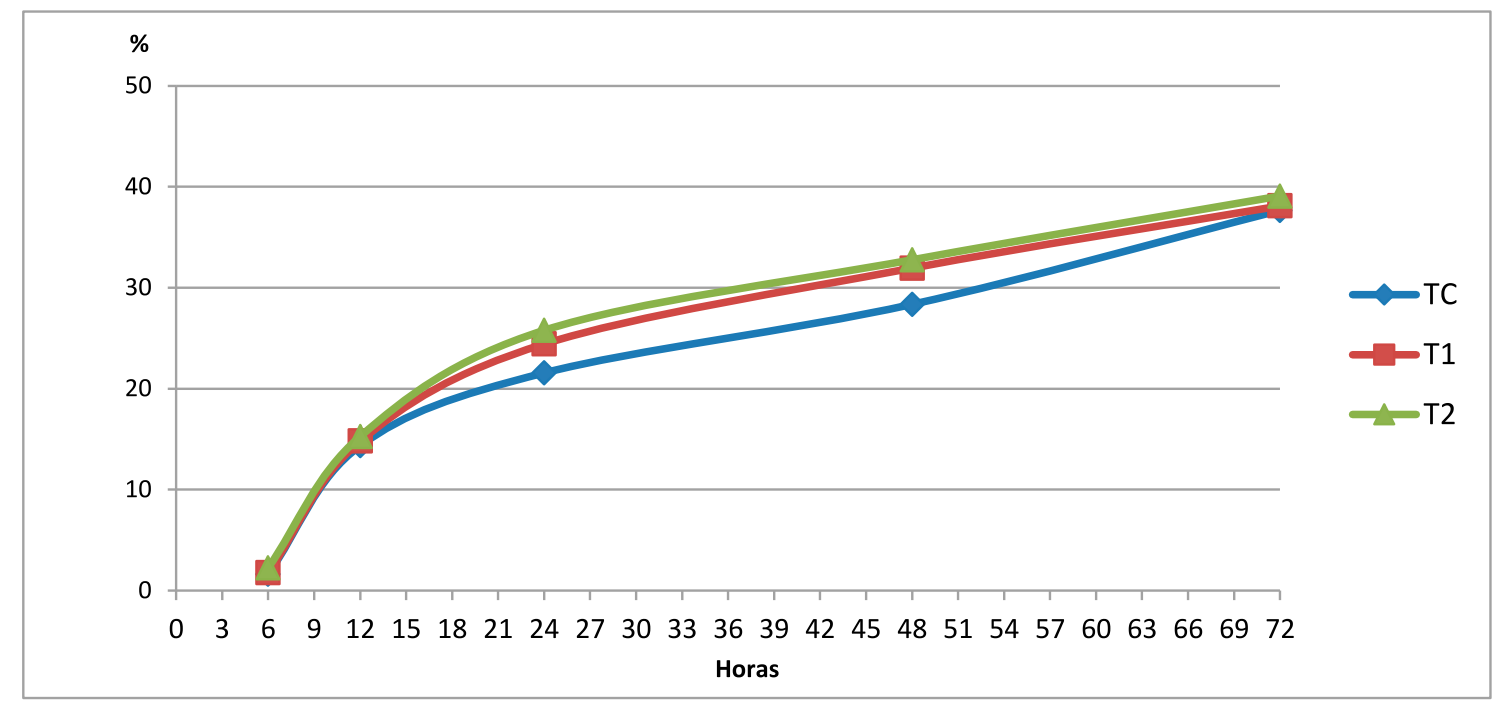

Fig. 1. Desaparición de la FDN (\%) en función del tiempo de incubación en rumen (horas). T=0 g Fibrozyme $^{\circledR} \mathrm{kg}_{\text {forraje }}{ }^{-1} \mathrm{MS} ; \mathrm{T} 1=1 \mathrm{~g}$ Fibrozyme $^{\circledR} \mathrm{kg}_{\text {forraje }}{ }^{-1} \mathrm{MS} ; \mathrm{T} 2=2 \mathrm{~g}^{\text {Fibrozyme }}{ }^{\circledR} \mathrm{kg}$ forraje ${ }^{-1} \mathrm{MS}$.

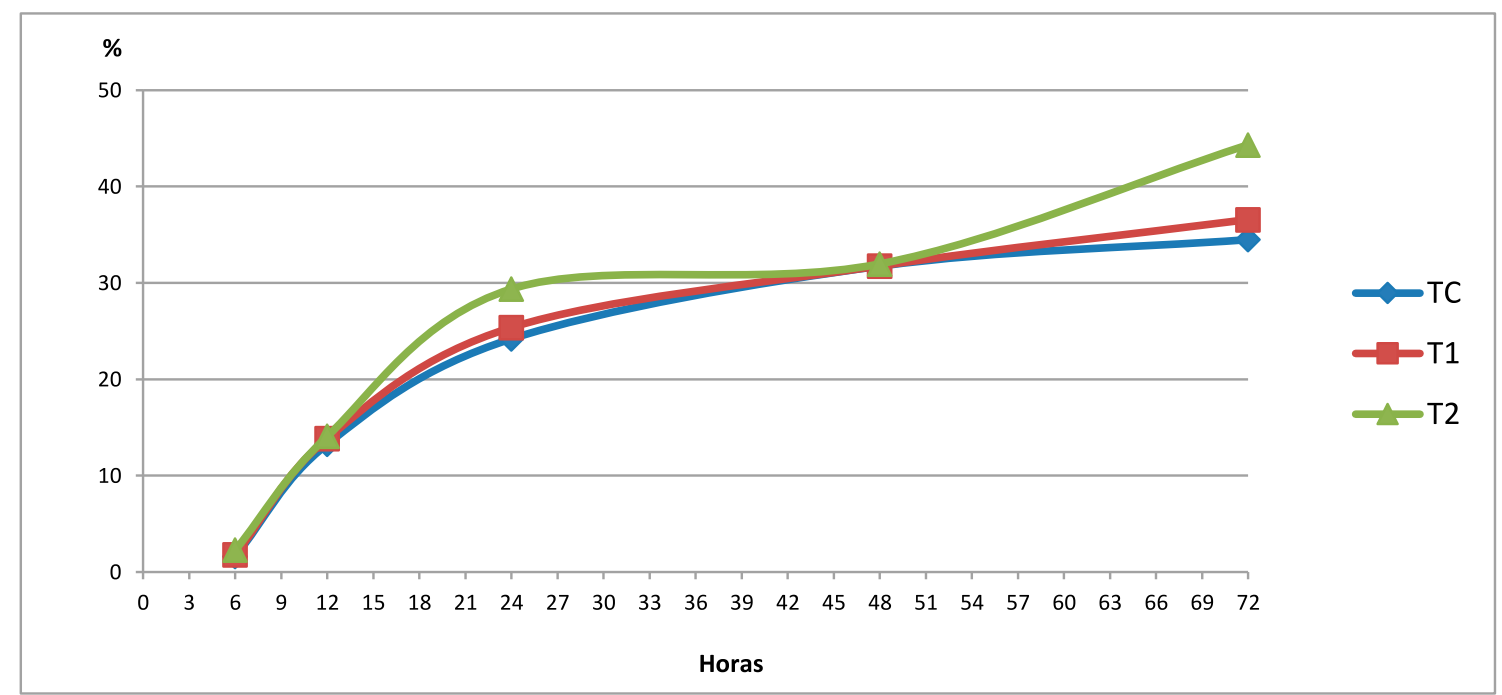

Fig. 2. Desaparición de la FDA (\%) en función del tiempo de incubación en rumen (horas). TC $=0 \mathrm{~g}$ Fibrozyme $^{\circledR} \mathrm{kg}_{\text {forraje }}{ }^{-1} \mathrm{MS} ; \mathrm{T} 1=1 \mathrm{~g}$ Fibrozyme ${ }^{\circledR} \mathrm{kg}$ forraje-1 MS; T2 = 2 g Fibrozyme $^{\circledR} \mathrm{kg}_{\text {forraje }}{ }^{-1} \mathrm{MS}$.

\section{Conclusión}

Los resultados de este experimento comprobaron que existe un efecto positivo del complejo enzimático Fibrozyme ${ }^{\circledR}$ (Alltech INC, Nicholasville, $\mathrm{KY}, \mathrm{USA}$ ) sobre la degradabilidad ruminal in situ de las fracciones de fibra (FDN y FDA) de rastrojo de maíz con el mayor nivel de la enzima (T2).

\section{Referencias}

(1) Macedo RJ. Análisis del sistema de alimentación pecuario rastrojo de maíz (Zea mayz L.) - pasto es- 
trella (Cynodon plectostachyus P.) en la zona norte del estado de Colima. Tesis de Doctorado, Facultad de Medicina Veterinaria y Zootecnia, Departamento de Posgrado Interinstitucional de Ciencia Pecuaria, Universidad de Colima. 2000.

(2) SAGARPA. Aprovechamiento de esquilmos y subproductos en la alimentación de ganado, 2009. [Consulta: 22 de octubre de 2013]. Disponible en: http://www.sagarpa.gob.mx/desarrolloRural/Documents/fichasaapt/Aprovechamiento $\% 20 \mathrm{de} \% 20$ esquilmos.pdf.

(3) Coronel U., Ortega ME, Mendoza G., Sánchez MT, Ayala TJ, Becerril C. Effect of two strains of Saccharomyces cerevisiae on productive performance of heifers. Nutrition Society. 2001.

(4) Chalupa W. Chemical control of rumen microbial metabolism. In: Digestive Physiology and Metabolism in Ruminants. Ruckebush Y., and P. Thivend (eds). Proceedings of the 5th International Symposium on Ruminant Physiology. MTP Press Limited, Lancaster, England. 1979; 325-347.

(5) Feng P., Hunt CW, Pritchard GT, Julien WE. Effect of enzyme preparations on in situ and in vitro degradation and in vivo digestive characteristics of mature cool - season grass forage in beef steers. J. Anim. Sci. 1996; 74: 1349-1357. DOI: http://doi. org/10.2527/1996.7461349x.

(6) Chen B. NEWAY EXCEL Versión 5.0. A utility for processing data of feed degradability and in vitro gas production. Rowett Research Institute. Aberdeen, Scotland. 1997.

(7) García E. Modificaciones al sistema de clasificación climática de Köppen (para adaptarlo a las condiciones de la República Mexicana). Offset Larios S.A. México D.F. 1988, 46-52.

(8) Paterson RT, Quiroga L., Sauma G., Samur C. Crecimiento de novillas Cebú Criollo en la época de seca con acceso limitado a la Leucaena. Prod. Anim. Trop. 1983; 8: 150.

(9) McDonald IM. A revised model for the estimation of protein degradability in the rumen. J. Agric. Sci. 1981; 96(1): 251-252. DOI: http://doi.org/10.1017/ s0021859600032081.
(10) Ørskov ER, McDonald IM. The estimation of protein degradability in the rumen from incubation measurements weighted according to rate of passage. J. Agric. Sci. 1979; 92(2): 499-503. DOI: http://doi.org/10.1017/S0021859600063048.

(11) Pedraza RM. Valoración nutritiva del follaje de Gliricidia sepium (Jacq.) kunth ex walp. y su efecto en el ambiente ruminal. Tesis Dr. Instituto de Ciencia Animal. La Habana, Cuba. 2000.

(12) AOAC. Official Methods of Analysis. 16th Ed. Ass. Off. Agric. Chem. Washington, DC. 1995.

(13) Van Soest PJ, Robertson JB, Lewis BA. Methods for dietary fiber, neutral detergent fiber, and nonstarch polysaccharides in relation to animal nutrition. J. Dairy Sci. 1991; 74(10): 3583-3597. DOI: http:// doi.org/10.3168/jds.S0022-0302(91)78551-2.

(14) Bourguetts LL. Efecto del tratamiento alcalino sobre la cinética de degradación ruminal de dos subproductos lignocelulosicos. Tesis de Maestría. Centro Universitario de Ciencias Biológicas y Agropecuarias. División Ciencias Veterinarias. Universidad de Guadalajara, México. 1998.

(15) Elizondo El. Evaluación de tratamientos alcalinos sobre la calidad nutricional de subproductos lignocelulósicos. Tesis de Doctorado. Facultad de Medicina Veterinaria y Zootecnia. Posgrado Interinstitucional en Ciencias Pecuarias Universidad de Colima, México. 1998.

(16) Estrada GT, Haro IM, Vázquez CRC, Martínez GDM, González DNT. Degradación in situ y patrones de fermentación del rastrojo de maíz (Zea mays L.) tratado con enzimas exógenas en vacas Holstein. Interciencia, 2015; 40(10): 716.

(17) Yescas R., Bárcena R., Mendoza G., González S., Cobos M., Ortega M. Digestibilidad in situ de dietas con rastrojo de maíz o paja de avena con enzimas fibrolíticas. Agrociencia, 2004; 38: 23-31.

(18) Salado EE, Comerón EA, Silva C., Gaggiotti MDC, Alesso A., Pardo J. Cascarilla de soja y afrechillo de trigo. Cinética de la degradabilidad ruminal de la fibra. Anuario EEA Rafaela. 2005. 